\title{
Addition of $\alpha-O$-GIcNAc to threonine residues define the post-translational modification of mucin-like molecules in Trypanosoma cruzi
}

\author{
Lucia Mendonça-Previato • Luciana Penha • \\ Tatiana Cortes Garcez • Christopher Jones • \\ Jose Osvaldo Previato
}

Received: 3 December 2012 /Revised: 3 February 2013 / Accepted: 6 February 2013 / Published online: 21 February 2013

(C) The Author(s) 2013. This article is published with open access at Springerlink.com

\begin{abstract}
Trypanosoma cruzi, an intracellular protozoan etiologic agent of Chagas disease is covered by a dense coat of mucin-type glycoproteins, which is important to promote the parasite entry and persistence in the mammalian host cells. The $O$-glycosylation of $T$. cruzi mucins (Tc-mucins) is initiated by enzymatic addition of $\alpha-O-N$-acetylglucosamine (GlcNAc) to threonine (Thr) by the UDP-GlcNAc: polypeptide $\alpha-N$-acetylglucosaminyltransferase (pp- $\alpha$ GlcNAcT) in the Golgi. The Tc-mucin is characterized by the presence of a high structural diversity of $O$-linked oligosaccharides found among different parasite strains, comprising two $O$-glycan Cores. In the Core 1 , from strains principally associated with the domestic transmission cycle of Chagas disease, the GlcNAc $O-4$ is substituted with a $\beta$ galactopyranose $(\beta \mathrm{Gal} p)$ unit, and in the most complex oligosaccharides the GlcNAc $O-6$ is further processed by the addition of $\beta 1 \rightarrow 2$-linked Gal $p$ residues creating a short linear Galp-containing chain. In the Core 2 structures, expressed by strains isolated from $T$. cruzi sylvatic hosts, the GlcNAc $O-4$ carries a $\beta$-galactofuranose $(\beta \mathrm{Gal} f)$ unit and the GlcNAc $O-6$ can carry a branched Galp $\beta 1 \rightarrow$ $3[\mathrm{Gal} p \beta 1 \rightarrow 2] \mathrm{Gal} p \beta 1 \rightarrow 6$ motif. The $O$-glycans carrying nonreducing terminal $\beta \mathrm{Gal} p$ are available for sialylation by a surface $T$. cruzi trans-sialidase activity. Based on
\end{abstract}

\footnotetext{
L. Mendonça-Previato $(\bowtie) \cdot$ L. Penha $\cdot$ T. C. Garcez $\cdot$

J. O. Previato

Laboratório de Glicobiologia, Instituto de Biofísica Carlos Chagas

Filho, Universidade Federal do Rio de Janeiro,

21941 902, Ilha do Fundão, Cidade Universitária,

Rio de Janeiro, RJ, Brazil

e-mail: luciamp@biof.ufrj.br

C. Jones

National Institute for Biological Standard and Control, Potters Bar, Hertfordshire EN6 3QG, UK
}

structural results, this review summarizes available data on the highly conserved process, which adds the GlcNAc unit in $\alpha$-linkage to Thr residues the basis of the post-translational modification system in T. cruzi mucins. In addition, a mechanism unique employed by the parasite to transfer exogenous sialic acid residues to Tc-mucins is presented.

Keywords Trypanosoma cruzi $\cdot$ Posttranslational modification $\cdot$ Mucins $\cdot$ pp- $\alpha$-GlcNAcT $\cdot$ trans-sialidase

\section{Introduction}

Chagas' disease, an infection caused by the protozoan Trypanosoma cruzi, remains a major cause of morbidity in Latin America. Though major advances in preventing the spread of this disease have been made in recent decades, an estimated 10 million people are infected due to prior exposure to T. cruzi [1], and about $30 \%$ of the individuals infected are characterized by heart inflammation and dysfunction [2]. T. cruzi presents genetic diversity, resulting in the prevalence of specific clinical forms and morbidity of Chagas disease, partially due to different protein expression levels and genomic instability [3]. Zingales and co-authors [4] subdivided T. cruzi species into six Discrete Typing Units (DTUs) designated $T$. cruzi I to T. cruzi VI. Recently, T. cruzi I has been correlated with cardiomyopathy manifestations $[5,6]$ increasing the need for further comparative biological and biochemical studies on different $T$. cruzi strains.

Protein glycosylation is an important post-translational modification underlying host-parasite interactions, which may determine the outcome of infection. The surface of $T$. cruzi is covered principally by a family of sialylglycoproteins (T. cruzi mucins) linked to the cell membrane through a 
glycosylphosphatidylinositol (GPI) anchor [7]. The protein domain is rich in threonine residues $[8,9]$ which can be modified with multiple $O$-linked glycan chains [10]. These $O$-glycans are acceptors of sialic acid derived from exogenous sialylglycoconjugates, through a reaction catalysed by a trypanosomal-specific trans-sialidase [11, 12]. The posttranslational modifications of $T$. cruzi mucins (Tc-mucin) give rise to $O$-linked glycans attached to the peptide by $\alpha$-GlcNAc$O$-Thr linkages [13], through the activity of a unique UDPGlcNAc:polypeptide $\alpha-N$-acetylglucosaminyltransferase (pp- $\alpha$-GlcNAcT) [13]. In contrast, in the mammalian mucins $O$-glycosylation, $N$-acetylgalactosamine (GalNAc) units are attached through $\alpha$-glycosidic linkage to the Ser and Thr residues [14-16].

The core $\alpha$-GlcNAc- $O$-Thr of Tc-mucins is further processed by $\beta$-galactopyranose ( $\beta$ Galp) (Core 1$)$ and $\beta$ galactofuranose $(\beta \mathrm{Gal} f)$ (Core 2$)$ units in a $T$. cruzi strainspecific pattern of linkages and substitutions $[10,17]$. Here we highlight the T. cruzi UDP-GlcNAc:polypeptide $\alpha-N$ acetylglucosaminyltransferase (pp- $\alpha$-GlcNAcT) and trans-sialidase activities, and unique $O$-glycan assemblies in Tc-mucins. Proven functions for the glycan domains of Tc-mucins on the pathogenesis of Chagas disease are unknown, although potential functions are addressed in this review. In fact, different strains of $T$. cruzi form a very heterogeneous group with specific characteristics such as histotropism, antigenicity, infectivity and pathogenicity [18], suggesting that the interaction of the parasite and human host cells would determine the severity of Chagas' disease. However, so far, the direct correlation of the structure of Tc-mucins O-glycans and the immunopathology of the disease has not been characterized.

\section{T. cruzi UDP-GlcNAc:polypeptide $\alpha$ - $N$-acetylglucosaminyltransferase (pp- $\alpha$-GlcNAcT)}

The post-translational modification of Tc-mucin with $O$-linked 2- $\mathrm{N}$-acetamido-2-deoxy-D-glucopyranose $(\mathrm{O}$-GlcNAc) is conserved in all T. cruzi strains studied to date (Fig. 1). Direct compositional analyses of Tc-mucin core proteins show that Thr are much more frequent than Ser residues [8, 9]. The same fact occurs in $T$. cruzi MUC gene-derived protein sequences [9] (Table 1). The $\alpha$-anomeric configuration of the protein- $O$ linked GlcNAc was determined by 2D-Nuclear Magnetic Resonance Spectroscopy (NMR) analysis of Smith-degraded sialylglycoproteins [13]. The key data were the ${ }^{3} J_{\mathrm{H} 1, \mathrm{H} 2}$ coupling constant, which is small, and ${ }^{1} J_{\mathrm{H} 1, \mathrm{Cl}}$, determined in an HSQC spectrum without ${ }^{13} \mathrm{C}$ decoupling. Both these techniques, combined with the chemical shift data and the resistance of the product of in vitro enzymatic GlcNAc addition to a synthetic peptide substrate $\left(\mathrm{KP}_{2} \mathrm{~T}_{8} \mathrm{KP}_{2}\right)$ to digestion with jack bean $\beta-N$ acetylglucosaminidase, indicate that the GlcNAc residue has the $\alpha$-anomeric configuration, thus distinguishing this system from the single $\beta$-linked GlcNAc residue observed as dynamic glycosylation on a number of nuclear and cytosolic proteins, and which is believed to serve a regulatory purpose $[19,20]$. The kinetic properties of the T. cruzi pp- $\alpha$-GlcNAcT were investigated using microsomal fractions prepared from insect-dwelling (epimastigotes) and cell-derived trypomastigote forms of T. cruzi II (DTU) [4] Y-strain, [7], the synthetic peptide acceptor KPPTTTTTTTTKPP, and with UDP- $\left[{ }^{3} \mathrm{H}\right]$ GlcNAc as the sugar donor $[13,21]$. The enzyme has an optimal $\mathrm{pH}$ of 7.5 to 8.5 and requires the presence of $\mathrm{Mn}^{2+}$. It is strongly inhibited by UDP but unaffected by the presence of tunicamycin or amphomycin, indicating that activated dolichol donor intermediates are not involved [13]. The microsome system from $T$. cruzi is unable to add $\left[{ }^{3} \mathrm{H}\right] \mathrm{GlcNAc}$ to the synthetic nonapeptide YSDSPSTST [22], the substrate for $O$ linked $\beta$ - $N$-acetylglucosamine transferase (OGT) an enzyme which catalyses a common post-translational modification of nuclear and cytoplasmic proteins $[19,20]$.

In $T$. cruzi a gene which encodes pp- $\alpha$-GlcNAcT activity was identified, designed TcOGNT-2 [23], and the predicted sequence is $61-81 \%$ similar to the 250 -amino-acid catalytic domain of DdGnt2, a Dictyostelium discoideum membranebound Golgi pp- $\alpha$ GlcNAcT $[24,25]$. Recently, it was demonstrated that TcOGNT-2 shows different levels of expression during the life cycle of $T$. cruzi. When trypomastigotes penetrate Vero cells and differentiate into amastigotes, TcOGnT-2 expression declines, and low levels of TcOGNT-2 mRNA and protein were detected. Later, when intracellular amastigotes differentiate in trypomastigotes the TcOGNT-2 increases expression again [26]. Interestingly, overexpressing of TcOGNT2 enhances T. cruzi infectivity [26].

\section{The assembly of Thr-linked $O$-glycans of Tc-mucins}

The assembly of Thr-linked $O$-glycans of Tc-mucins is initiated in the Golgi $[13,21]$ by the pp- $\alpha$-GlcNAcT. The $O-\alpha$-GlcNAc residues are further processed to form the backbone structure for biologically important epitopes. The addition of galactopyranose and galactofuranose monosacharides is catalyzed by $\beta 1,6 ; \beta 1,4 ; \beta 1,3 ; \beta 1,2-\mathrm{Gal} p$ transferases and $\beta 1,4 ; \beta 1,2-\mathrm{Gal} f$ transferases, and the attachment of sialic acid at some terminal $\beta \mathrm{Gal} p$ residues is catalyzed by a trans-sialidase activity [7].

The simplest glycosylation pattern found in Tc-mucin consists of a single unsubstituted $O$-linked GlcNAc residue. In the T. cruzi Tulahuen strain [27], high performance liquid chromatography (HPLC) analyses suggest that single GlcNAc residues are present at about $20 \%$ of the glycosylation sites, and similarly high amounts are present in the $O$ glycan mixture from other strains [7, 8, 17, 28, 29] (Fig. 1). $O$-glycans were isolated as alditols from purified Tc-mucins by alkaline reductive cleavage and fractionated by gel 


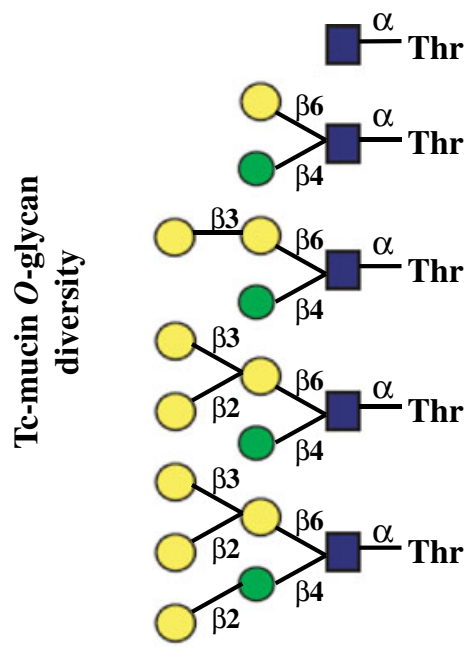

G [8] and Colombiana strains [17] T. cruzi I [4]
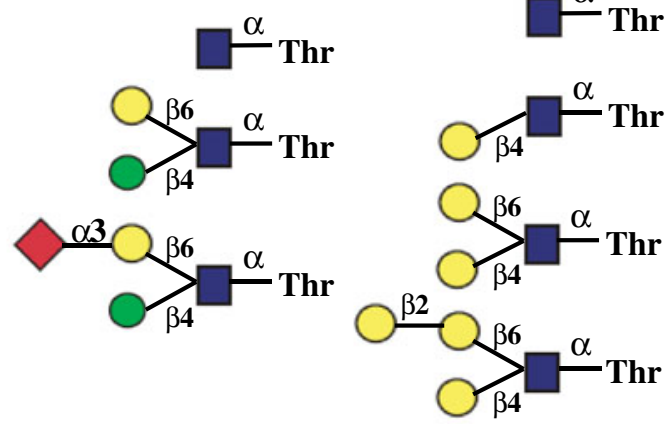

Y strain [7]

T. cruzi II [4]

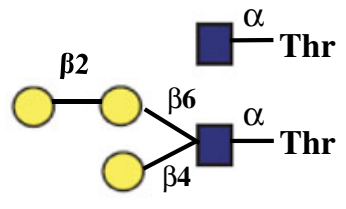

$\overbrace{\beta 4}^{\beta 6}{ }^{\alpha} \mathbf{T h r}$

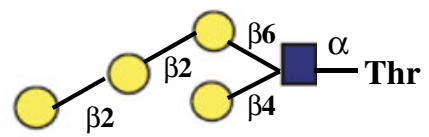

CL-Brener strain [29]

T. cruzi VI [4]
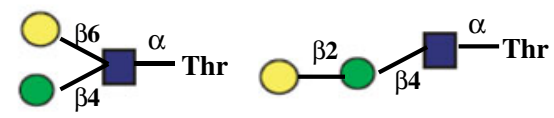

ฮี่
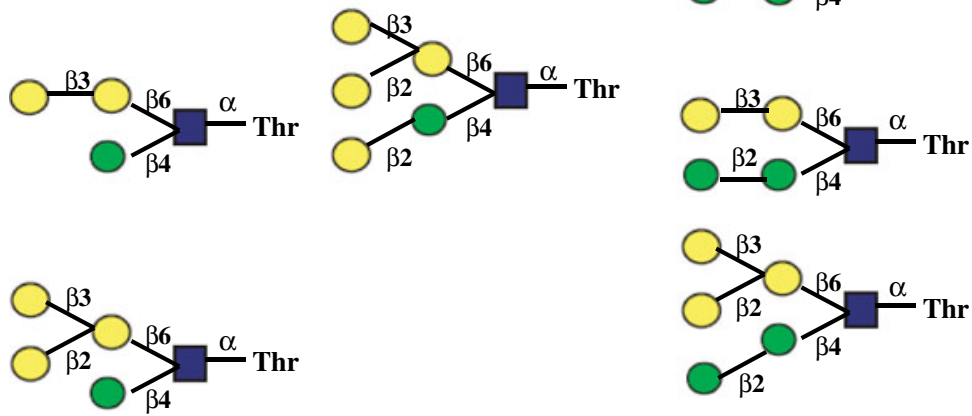

Tulahuen strain [27]

T. cruzi VI [4]

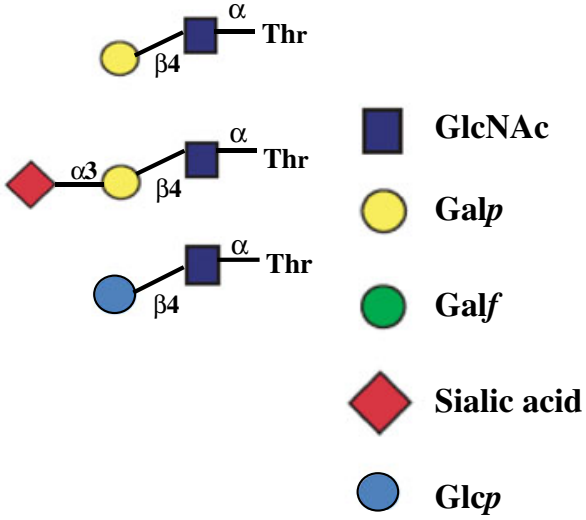

GIcNAc

Galp

Galf

Glcp

Fig. 1 The structures of the derived glycan of -O-Thr Tc-mucins from different T. cruzi strains. Representative glycan structure is indicative with colored geometric symbols, conform to those recommended by Consortium for Functional Glycomics

filtration chromatography [8]. The purification is difficult due to high levels of glycosylation with eventual sialylation, thus when it is required further purification by HPLC on pyrolysed graphitic carbon (PGC) was performed [27-29]. $O$-glycan chemical structures from Tc-mucins were determined, usually, by one- and two-dimensional (2D) homo- and hetero-nuclear NMR spectroscopy combined with methylation analysis, mild acid hydrolysis and mass spectrometry.

Two options for the addition of the first Gal residue have been identified. The Core 1, in the Y [7] and CL-Brener [29] strains, a $\beta \mathrm{Gal} p$ residue is transferred to the GlcNAc $O-4$ (Fig. 1). The core 2, in the G [8], Colombiana [17], and Dm28c [28] strains, a $\beta \mathrm{Gal} f$ residue is attached at GlcNAc $O-4$ to give structures shown in Fig. 1. The $O$-glycans from $T$. cruzi Tulahuen strain mucins show high structure diversity
[27]. The Cores 1 and 2 are synthesized by Tulahuen strain, the Gal $p \beta \rightarrow 4$ GlcNAc (Core 1) and Gal $\beta \beta \rightarrow 4$ GlcNAc (Core 2). Surprisingly, sialylation is the only observed elaboration of the Galp $\beta \rightarrow 4$ GlcNAc [27] (Fig. 1). In the Tulahuen strain [27] besides both Galp $\beta \rightarrow 4$ GlcNAc $\alpha \rightarrow$ Thr and Gal $\beta \rightarrow$ 4GlcNAc $\alpha \rightarrow$ Thr there is also evidence for $O$-glycan with a $\beta$-glucose residue on GlcNAc $O-4$. This appears to be a "dead-end" species, as no higher oligosaccharides with this glucosylation pattern have been observed.

\section{Extension of the GlcNAc $\alpha 1-/ T h r$}

In the $\mathrm{CL}$ and $\mathrm{Y}$ T. cruzi strains, more complex glycan structures arise from the attachment of a $\beta$-Gal $p$ residue at GlcNAc $O-6$, leading to a disubstituted oligosaccharide (Fig. 1). Further 
Table 1 Amino acid composition of Tc-mucins purified from different T. cruzi strains

\begin{tabular}{|c|c|c|c|c|c|}
\hline \multirow[t]{2}{*}{${ }^{\mathrm{a} A m i n o ~ a c i d}$} & \multicolumn{5}{|c|}{ T. cruzi strain } \\
\hline & G & $\mathrm{Y}$ & CL-Brener & Tulahuen & ${ }^{\mathrm{b}} \mathrm{MUC1} 1-\mathrm{Y}$ [9] \\
\hline Thr & 50.3 & 45.9 & 53.8 & 50.5 & 54.5 \\
\hline Asp/Asn & 12.8 & 11.2 & 10.0 & 12.9 & 4.5 \\
\hline Ala & 10.3 & 10.1 & 8.7 & 10.6 & 5.7 \\
\hline Pro & 9.3 & 8.4 & 7.8 & 6.3 & 11.4 \\
\hline Ser & 0.9 & 1.1 & 0.9 & 0.9 & 4.6 \\
\hline Glu/Gln & 5.2 & 6.9 & 5.4 & 5.7 & 5.7 \\
\hline Lys & 0.6 & 4.4 & 0.6 & 2.1 & 3.4 \\
\hline Gly & 7.1 & 8.5 & 6.8 & 7.8 & 0.0 \\
\hline Arg & 0.0 & 0.4 & 0.3 & 0.0 & 4.6 \\
\hline Val & 2.3 & 1.6 & 3.2 & 2.3 & 1.1 \\
\hline Met & 0.0 & 0.0 & 0.0 & 0.0 & 0.0 \\
\hline Ile & 0.4 & 0.7 & 0.8 & 0.3 & 3.4 \\
\hline Tyr & 0.0 & 0.0 & 0.0 & 0.0 & 0.0 \\
\hline Leu & 0.5 & 0.5 & 0.5 & 0.5 & 0.0 \\
\hline His & 0.4 & 0.3 & 0.5 & 0.0 & 0.0 \\
\hline Phe & 0.0 & 0.0 & 0.0 & 0.0 & 0.0 \\
\hline
\end{tabular}

${ }^{a}$ Amino acid content is indicated in number of residues per 100 residues

${ }^{\mathrm{b}}$ Composition deduced from the gene sequence

elaborations occur by addition of one or two $\beta$-Galp $p(1 \rightarrow 2)$ residues to the Gal $p$ present on the GlcNAc $O-6$, thus a short linear galactan chain is created on the 6-arm [27, 29]. Significant in the Core 1 structure is the presence of $O$-glycans terminated by Galp $\alpha 1 \rightarrow 3$. In Tc-mucins isolated from cellderived trypomastigotes the $O$-glycans contain the trisaccharide Galp $\alpha 1 \rightarrow 3 \mathrm{Gal} p \beta \rightarrow 4 \mathrm{GlcNAc} \alpha \rightarrow[30]$. The $\alpha$ galactosylated glycans are highly immunogenic to humans and represent the major epitope for trypanolytic anti- $\alpha \mathrm{Gal}$ antibodies found in the serum of acute and chronic chagasic patients $[30,31]$.

Three biosynthetic pathways by which Core 2 Gal $\beta \rightarrow$ 4GlcNAc $\alpha \rightarrow$ are elaborated and have been characterized [27]. The first is similar to that seen in the Core 1, with addition of a $\beta \mathrm{Gal} p$ residue to the GlcNAc $O-6$, to give rise to disubstituted structure (Fig. 1). This has been observed for the G-, Colombiana-, Tulahuen- and Dm28c- strains [8, $17,27,28]$. Second, in the Tulahuen strain, glycans resulting from the addition of either $\beta$-Gal $p 1 \rightarrow 2$ or $\beta-G a l f \beta 1 \rightarrow 2$ to the Galf $\beta \rightarrow 4$ GlcNAc $\alpha \rightarrow$ are observed, leading to approximately equal amounts [27]. Third, the expression of Gal $f \beta 1 \rightarrow 2 \mathrm{Gal} f \beta \rightarrow 4 \mathrm{GlcNAc} \alpha \rightarrow$ motif appears to be unique in Tc-mucins. Mammalian cells do not produce glycoproteins or glycolipids containing Galf, and this epitope elicits a strong immune response in $T$. cruzi infected mice [32].
The $O$-glycans containing both $\beta$-Gal $f$ and $\beta$-Gal $p$ linked to $\rightarrow 4$ GlcNAc $\alpha \rightarrow$ and $\rightarrow 6$ GlcNAc $\alpha \rightarrow$, respectively, have terminal $\beta$-Gal $p$ residues available for sialylation. Along this line, the Tc-mucins from Dm28c [28] and Tulahuen [27] strains express $O$-glycan containing as nonreducing end, both sialic acid and Galf residues (Fig. 1). Using synthetic Gal $f$ and Galp-contaning oligosaccharides, with a recombinant preparation of T. cruzi trans-sialidase, and sialyllactose as sialic donor, Agustí and co-authors [33] verified that the presence of Gal $f$ in the O-glycans from Tc-mucins does not impair their acceptor properties. Furthermore, this third biosynthetic pathway forms a trigalactosylated (Fig. 1, G-, Colombiana, Dm28c, Tulahuen strains) glycan, which differs from glycan in Family 1 in that the additional Gal $p$ residue is linked $\beta 1 \rightarrow 3$ rather than $\beta 1 \rightarrow 2$; also, two tetragalactosylated members, which the most common arises by addition of a Galp $\beta 1 \rightarrow 2$ to the 3substituted Gal $p$ residue attached to the GlcNAc $O-6$. The presence of a branched tri-galactose structure on the 6-arm appears to be restricted to Core 2. A minor tetragalactosylated glycan (Fig. 1, Tulahuen strain) arises by addition of a $\beta$-Gal $f$ unit to the Gal $f O-2$. Two pentagalactosylated structures have been also observed, arising by addition of either a $\beta$-Galp or a $\beta$-Gal $f$ residue to $O-2$ of the Gal $f$ on the GlcNAc $O-4$, resulting in structures described in Fig. 1 (Tulahuen strains). These data imply the presence of different $\beta$-galactopyranosyl transferases expressed in a straindependent manner, emphasizing that the Tc-mucin $O$-glycan structures are strain-dependent.

Sialylation of the $T$. cruzi $O$-linked glycans occurs through the action of a parasite-specific trans-sialidase [11], which transfers sialic acid from Neu5Ac $\alpha 2 \rightarrow$ $3 \mathrm{Gal} p \beta$-containing exogenous donor molecules to terminal $\beta \mathrm{Gal} p$-containing acceptors, attaching it in an $\alpha 2-3$ linkage configuration. A mixture of anionic oligosaccharides was isolated from the Tc-mucins of CL-Brener strain [29], which were characterized as $3^{\prime}$-sialyl lactosaminitol, Neu5Ac $\alpha 2 \rightarrow$ 3Gal $p \beta 1-4 \mathrm{GlcNAc} \alpha 1$ - and two 3'-monosialylated variants, $\mathrm{Gal} p \beta 1 \rightarrow 4[\mathrm{Gal} p \beta 1 \rightarrow 6] \mathrm{GlcNAc}$, in approximately, equal amounts, suggesting $T$. cruzi trans-sialidase has no specificity for the 4- or 6- arm. Although all terminal $\beta \mathrm{Gal} p$ residues are potential acceptors for sialic acid, no sialylated forms of the more complex Core 2 glycans have been observed, and so any selectivity in the sialylation of the various nonreducing end $\beta$-Gal $p$ residues remains undefined. Also, no evidence was found for disialylated $O$-glycans. Consistent with data from in vitro sialylation of $O$ linked glycans purified from Tc-mucins of epimastigotes [7] and metacyclic trypomastigotes [34]. The incorporation of one molecule of sialic acid hinders entry of a second molecule when two potential acceptor sites are present. The $T$. cruzi trans-sialidase substrate donor specificity has been the subject of research for many groups $[35,36]$, likewise there 
are different patented processes related to the enzymatic synthesis of sialyl $\alpha 2 \rightarrow 3 \beta$ galactosides, using this enzyme.

\section{T. cruzi trans-sialidase (Tc-TS) activity and Tc-mucins}

Four points related with the pathogenesis of Chagas disease are importants: (i) Tc-mucins are the main acceptors of sialic acid in trans-sialidase mediated reaction [7, 8, 37]; (ii) sialylation of Tc-mucin $O$-glycans is crucial for the viability and persistence of $T$. cruzi in mammalian hosts [38-40]; (iii) the initial incorporation of GlcNAc through pp- $\alpha$-GlcNAcT is a limiting step for the addition of sialic acid by T. cruzi trans-sialidase (Tc-TS); and (iv) no similar mammalian enzymes were described.

Hundreds of genes encoding Tc-TS enzymes and Tcmucin glycoproteins are present in the T. cruzi genome, and, interestingly, Tc-mucins glycoproteins genes are closely linked to members of the trans-sialidase super-family at multiple sites in the $T$. cruzi genome [41]. The co-expression of TcTS and pp- $\alpha$-GlcNAcT has been also observed [26]. Furthermore, there are evidences that the increase or decrease of Tc-TS and pp- $\alpha$-GlcNAcT expressions are dependent upon the different forms of the parasite, during the infectious process [26].

The T. cruzi TS is an enzyme located on the external surface of the parasite, and a modified sialidase that, instead of releasing sialic acid, can transfer the host-derived sialic acid to terminal $\beta \mathrm{Gal} p$ in the Tc-mucin $O$-glycans. This enzymatic reaction is different from the known sialyltransferases present in the Golgi that exclusively use CMP-sialic acid as the donor substrate.

The first evidence on a novel pathway for the incorporation of sialic acid into T. cruzi glycoproteins, through an unusual transglycosylase activity, was done by Previato and co-authors [11]. The authors have observed that T. cruzi cells grown in the presence of fetal calf serum (sialic acid donor) were agglutinated by wheat germ agglutinin (WGA), a lectin that also recognizes terminal sialic acid units. Nonetheless, in the absence of fetal calf serum in the medium culture, or if the parasites were treated with Clostridium perfringes neuraminidase, the WGA binding was abolished and instead the T. cruzi cells agglutinated by peanut agglutinin (PNA), a lectin that recognizes terminal residues of $\beta \mathrm{Gal} p$. Further, these later cells regained their WGA agglutinability when incubated with fetuin or sialyllactose, but not with free sialic acid. These same results were obtained with energy-rich and energy-depleted T. cruzi cells [11]. Later, the presence of trans-sialidase activity was proven, and established that the expression of TcTS and the acquisition of sialic acid by $T$. cruzi are relevant events in the interaction and invasion of the parasite to the host $[38,42]$.

\section{Developmental life cycle of $T$. cruzi and Tc mucin functions}

T. cruzi presents a complex life cycle involving the hematophagous triatomine insect and mammalian host species, with different developmental stages. Within the insect, T. cruzi differentiates in two diverse forms: replicative epimastigote and non-replicative metacyclic trypomastigote forms. Metacyclic trypomastigotes are mostly transmitted during a blood meal of the insect, which are able to invade a wide variety of mammalian nucleated cells. In mammalian hosts, T. cruzi behaves as an obligate intracellular pathogen. Inside the cell, the infective trypomastigotes are temporarily contained in the parasitophorous vacuole subsequently the parasites escape to the cytosol, and differentiate into the replicative amastigotes, which after several divisions, transform into cell-derived trypomastigotes, which are released into the bloodstream. The T. cruzi life cycle closes when a triatomine vector feeds on a $T$. cruzi-infected mammal [43]. Several mechanisms of infection have been proposed for the extremely complex $T$. cruzi-host cell interaction process, which involves many putative $T$. cruzi ligands and a growing list of host cell targets [44, 45].

Here, we summarize the main aspects of $T$. cruzi-host cells interactions, involving Tc-mucins. Tc-mucins from epimastigotes and metacyclic trypomastigotes differ from those of cell culture trypomastigotes in their apparent molecular masses. The Tc-mucins isolated from T. cruzi insect forms migrate on SDS-PAGE as a broad band in 35-50 $\mathrm{kDa}$ range $[8,11]$, while Tc-mucins from cell-derived trypomastigotes present a wide range from 60 to $200 \mathrm{kDa}$ molecular masses [30, 42], these are equivalent to the highly glycosylated protein sharing sialic acid-containing epitopes crucial for mammalian cell attachment and invasion [42]. Despite the relevance of cell-derived trypomastigotes, little is known about the chemical structure of $O$-glycans of Tc-mucins from these forms, however a key difference from the insect-stage mucins, is the presence of terminal $\alpha$-galactopyranosyl residues, which are targets of lytic antibodies isolated from patients with chronic Chagas disease, in cell-derived trypomastigote mucins [30, 31]. Tc-mucins from cell-derived trypomastigotes induce the synthesis of nitric acid and proinflammatory cytokines IL- 12 and TNF- $\alpha$ by stimulated macrophages [46], effects that may be modulate the immune response to $T$. cruzi during the infection. Many of the biological properties of Tc-mucins have been related to the presence of sialic acid-containing $\alpha$-O-linked glycans [42]. It has been demonstrated that Tc-mucins are the main acceptors of sialic acid in trans-sialidase mediated reaction, and that the sialylation of Tc-mucin O-glycans is crucial for the viability and persistence of these parasites. Nevertheless, Yoshida and co-author [47] demonstrated that the ability of cell-derived trypomastigotes, obtained from $T$. cruzi G-strain, to invade HeLa cells is independent of sialic acid, providing evidence that the rate of invasion of desialylated parasites is significantly 
higher after treatment of both $T$. cruzi and purified Tc-mucin with neuraminidase. However, this effect seems to be straindependent. The major structural features do not differ between $O$-glycans from epimastigote [7] and metacyclic trypomastigote forms [34], however, mucins from metacyclic trypomastigotes, but not from epimastigotes, bind to cultured cell lines [48]. The involvement of Tc-mucins from metacyclic trypomastigotes, in invasion to mammalian cell lines, was verified by inhibition of parasite internalization by monoclonal antibodies that recognize Gal $p$ or Galf-containing epitopes of $O$ glycans [49]. The mechanism of interaction of metacyclic trypomastigotes-host cells mainly relies on $T$. cruzi strains which express on their surface variant forms of $O$-glycan and exhibit diverse range of capability to invade host cells in vitro $[50,51]$.

\section{Conclusion}

The surface of $T$. cruzi is covered by mucin-like molecules (Tcmucins) which are implicated to parasite protection in both vertebrate and invertebrate hosts, in mechanisms of infectivity and modulation of the host immune response throughout the $T$. cruzi life cycle. The obvious medical significance of $T$. cruzi and the knowledge of the molecular structure of the Tc-mucin have led to intensive study of its biosynthesis. The first step of $O$-glycosylation of Tc-mucins is a unique biosynthetic pathway catalyzed by a pp- $\alpha$-GlcNAcT, which transfers $\alpha-O-N$ acetylglucosamine (GlcNAc) to threonine (Thr) residues.

The pathways leading to $O$-glycosylation of $T$. cruzi glycoproteins show unusual features when compared to that of mammalian cells. Optimistically, the selective expression of enzymes, which are not present in the parasite's hosts, such as the pp- $\alpha$-GlcNAcT and TcTS, an enzyme with a unique specificity for the addition of sialic acid on Tc-mucins, might provide suitable novel targets for the development of less toxic and more effective treatments against Chagas' disease.

Acknowledgments The authors kindly acknowledge the Conselho Nacional de Ciência e Tecnologia (CNPq), Fundação de Auxílio à Pesquisa do Estado do Rio de Janeiro (FAPERJ), Coordenação de Aperfeiçoamento de Pessoal de Nível Superior (CAPES).

Open Access This article is distributed under the terms of the Creative Commons Attribution License which permits any use, distribution, and reproduction in any medium, provided the original author(s) and the source are credited.

\section{References}

1. WHO: Chagas disease (American trypanosomiasis). Fact sheet $\mathbf{3 4 0}$ (2010)

2. Machado, F.S., Jelicks, L.A., Kirchhoff, L.V., Shirani, J., Nagajyothi, F., Mukherjee, S., Nelson, R., Coyle, C.M., Spray, D.C., de Carvalho, A.C., Guan, F., Prado, C.M., Lisanti, M.P., Weiss, L.M.,
Montgomery, S.P., Tanowitz, H.B.: Chagas heart disease: report on recent developments. Cardiol. Rev. 20(2), 53-65 (2012)

3. Bellini, M.F., Silistino-Souza, R., Varella-Garcia, M., de AzeredoOliveira, M.T., Silva, A.E.: Biologic and genetics aspects of Chagas disease at endemic areas. J. Trop. Med. (2012). doi:10.1155/2012/357948. Article ID 357948

4. Zingales, B., Andrade, S.G., Briones, M.R., Campbell, D.A., Chiari, E., Fernandes, O., Guhl, F., Lages-Silva, E., Macedo, A.M., Machado, C.R., Miles, M.A., Romanha, A.J., Sturm, N.R., Tibayrenc, M., Schijman, A.G.: Second satellite meeting. A new consensus for Trypanosoma cruzi intraspecific nomenclature: second revision meeting recommends TcI to TcVI. Mem. Inst. Oswaldo Cruz 104(7), 1051-1054 (2009)

5. Ramírez, J.D., Guhl, F., Rendón, L.M., Rosas, F., Marin-Neto, J.A., Morillo, C.A.: Chagas cardiomyopathy manifestations and Trypanosoma cruzi genotypes circulating in chronic Chagasic patients. PLoS Negl. Trop. Dis. 4(11), e899 (2010)

6. Guhl, F., Ramírez, J.D.: Trypanosoma cruzi I diversity: towards the need of genetic subdivision? Acta Trop. 119(1), 1-4 (2011)

7. Previato, J.O., Jones, C., Xavier, M.T., Wait, R., Travassos, L.R., Parodi, A.J., Mendonça-Previato, L.: Structural characterization of the major glycosylphosphatidylinositol membrane anchored glycoprotein of epimastigote forms of Trypanosoma cruzi Y-strain. J. Biol. Chem. 270(13), 7241-7250 (1995)

8. Previato, J.O., Jones, C., Gonçalves, L.P., Wait, R., Travassos, L.R., Mendonça-Previato, L.: $O$-Glycosidically linked Nacetylglucosamine-bound oligosaccharides from glycoproteins of Trypanosoma cruzi. Biochem. J. 301(Pt1), 151-159 (1994)

9. Di Noia, J.M., Pollevick, G.D., Xavier, M.T., Previato, J.O., Mendoça-Previato, L., Sánchez, D.O., Frasch, A.C.: High diversity in mucin genes and mucin molecules in Trypanosoma cruzi. J. Biol. Chem. 271(50), 32078-32083 (1996)

10. Mendonça-Previato, L., Todeschini, A.R., Heise, N., Agrellos, O.A., Previato, J.O.: Chemical structure of major glycoconjugates from parasites. Curr. Org. Chem. 12(11), 926-939 (2008)

11. Previato, J.O., Andrade, A.F.B., Pessolani, M.C.V., Mendonça Previato, L.: Incorporation of sialic acid into Trypanosoma cruzi macromolecules - a proposal for a new metabolic route. Mol. Biochem. Parasitol. 16(1), 85-96 (1985)

12. Mendonça-Previato, L., Todeschini, A.R., Freire de Lima, L., Previato, J.O.: The trans-Sialidase from Trypanosoma cruzi a Putative Target for Trypanocidal Agents. Open Parasitol. J. 4, 111-115 (2010)

13. Previato, J.O., Sola-Penna, M., Agrellos, O.A., Jones, C., Oeltmann, T., Travassos, L.R., Mendonça-Previato, L.: Biosynthesis of $O$ - $N$-acetylglucosamine-linked glycans in Trypanosoma cruzi: characterisation of the novel uridine diphospho- $N$-acetylglucosamine:polypeptide $N$-acetyl-glucosaminyl transferase catalysing formation of the $\alpha-N$-acetylglucosamine $(1 \rightarrow O)$-threonine linkage. J. Biol. Chem. 273(24), 1498214988 (1998)

14. Ten Hagen, K.G., Fritz, T.A., Tabak, L.A.: All in the family: the UDP-GalNAc:polypeptide N-acetylgalactosaminyltransferases. Glycobiology 13(1), 1R-16R (2003)

15. North, S.J., Hitchen, P.G., Haslam, S.M., Dell, A.: Mass spectrometry in the analysis of N-linked and O-linked glycans. Curr. Opin. Struct. Biol. 19(5), 498-506 (2009)

16. Cullen, P.J.: Post-translational regulation of signaling mucins. Curr. Opin. Struct. Biol. 21(5), 590-596 (2011)

17. Todeschini, A.R., de Almeida, E.G., Agrellos, O.A., Jones, C., Previato, J.O., Mendonça-Previato, L.: Alpha-N-acetylglucosaminelinked O-glycans of sialoglycoproteins (Tc-mucins) from Trypanosoma cruzi Colombiana strain. Mem. Inst. Oswaldo Cruz 104(Suppl 1), 270-274 (2009) 
18. Miles, M.A., Souza, A., Povoa, M., Shaw, J.J., Lainson, R., Toye, P.J.: Isozymic heterogeneity of Trypanosoma cruzi in the first autochthonous patients with Chagas' disease in Amazonian Brazil. Nature (London) 272, 819-821 (1978)

19. Slawson, C., Hart, G.W.: O-GlcNAc signalling: implications for cancer cell biology. Nat. Rev. Cancer 11(9), 678-684 (2011)

20. Zachara, N.E., Vosseller, K., Hart, G.W.: Detection and analysis of proteins modified by O-linked $\mathrm{N}$-acetylglucosamine. Curr. Protoc. Protein Sci. 66(12.8), 1-12 (2011)

21. Morgado-Díaz, J.A., Nakamura, C.V., Agrellos, O.A., Dias, W.B., Previato, J.O., Mendonça-Previato, L., De Souza, W.: Isolation and characterization of the Golgi complex of the protozoan Trypanosoma cruzi. Parasitology 123(Pt1), 33-43 (2001)

22. Haltiwanger, R.S., Holt, G.D., Hart, G.W.: Enzymatic addition of $O$-GlcNAc to nuclear and cytoplasmic proteins - identification of uridine diphospho- $N$-acetylglucosamine peptide $\beta-N$ acetylglucosaminyltransferase. J. Biol. Chem. 265(5), 25632568 (1990)

23. Heise, N., Singh, D., van der Wel, H., Sassi, S.O., Johnson, J.M., Feasley, C.L., Koeller, C.M., Previato, J.O., Mendonça-Previato, L., West, C.M.: Molecular analysis of a UDP-GlcNAc:polypeptide alpha-N-acetylglucosaminyltransferase implicated in the initiation of mucin-type O-glycosylation in Trypanosoma cruzi. Glycobiology 19(8), 918-933 (2009)

24. Wang, F., Metcalf, T., van der Wel, H., West, C.M.: Initiation of mucin-type O-glycosylation in dictyostelium is homologous to the corresponding step in animals and is important for spore coat function. J. Biol. Chem. 278(51), 51395-51407 (2003)

25. West, C.M., Van Der Wel, H., Sassi, S., Gaucher, E.: Cytoplasmic glycosylation of protein-hydroxyproline and its relationship to other glycosylation pathways. Biochem. Biophys. Acta 1673(12), 29-44 (2004)

26. Chiribao, M.L., Libisch, M.G., Osinaga, E., Parodi-Talice, A., RobelloCloning, C.: Localization and differential expression of the Trypanosoma cruzi TcOGNT-2 glycosyl transferase. Gene 498(2), 147-154 (2012)

27. Jones, C., Todeschini, A.R., Agrellos, O.A., Previato, J.O., Mendonça-Previato, L.: Heterogeneity in the biosynthesis of mucin $O$-glycans from Trypanosoma cruzi Tulahuen strain with the expression of novel galactofuranosyl-containing $\alpha-O$-GlcNAclinked oligosaccharides. Biochemistry 43(37), 11889-11897 (2004)

28. Agrellos, O.A., Jones, C., Previato, J.O., Mendonça-Previato, L.: A novel sialylated and galactofuranose-containing $O$-linked glycan, Neu5Ac $\alpha 2 \rightarrow \mathrm{Gal} p \beta 1 \rightarrow 6(\mathrm{Gal} f \beta 1 \rightarrow 4)$ GlcNAc, is expressed on the sialoglycoprotein of Trypanosoma cruzi Dm28c. Mol. Biochem. Parasitol. 126(1), 93-96 (2003)

29. Todeschini, A.R., Xavier da Silveira, E., Jones, C., Wait, R., Previato, J.O., Mendonça-Previato, L.: Structures of $O$ Glycosidically linked oligosaccharides from glycoproteins of Trypanosoma cruzi CL-Brener strain: Evidence for the presence of $O$-linked sialyloligosaccharides. Glycobiology 11(1), 47-55 (2001)

30. Almeida, I.C., Ferguson, M.A.J., Schenkman, S., Travassos, L.R.: Lytic anti- $\alpha$-galactosyl antibodies from patients with chronic Chagas' disease recognize novel $O$-linked oligosaccharides on mucin-like glycosylphosphatidylinositol-anchored glycoproteins of Trypanosoma cruzi. Biochem. J. 304(3), 793-802 (1994)

31. Pereira-Chioccola, V.L., Acosta-Serrano, A., Almeida, I.C., Ferguson, M.A., Souto-Padron, T., Rodrigues, M.M., Travassos, L.R., Schenkman, S.: Mucin-like molecules form a negatively charged coat that protects Trypanosoma cruzi trypomastigotes from killing by human anti-alpha-galactosyl antibodies. J. Cell Sci. 113(7), 1299-1307 (2000)

32. Mendonça-Previato, L., Gorin, P.A., Braga, A.F., Scharfstein, J., Previato, J.O.: Chemical structure and antigenic aspects of complexes obtained from epimastigotes of Trypanosoma cruzi. Biochemistry 22(21), 4980-4987 (1983)

33. Agustí, R., Giorgi, M.E., Mendoza, V.M., Gallo-Rodriguez, C., de Lederkremer, R.M.: Comparative rates of sialylation by recombinant trans-sialidase and inhibitor properties of synthetic oligosaccharides from Trypanosoma cruzi mucins-containing galactofuranose and galactopyranose. Bioorg. Med. Chem. 15(7), 2611-2616 (2007)

34. Serrano, A.A., Schenkman, S., Yoshida, N., Mehlert, A., Richardson, J.M., Ferguson, M.A.J.: The lipid structure of the glycosylphosphatidylinositol-anchored mucin-like sialic-acid acceptors of Trypanosoma cruzi changes during parasite differentiation from epimastigotes to infective metacyclic trypomastigote forms. J. Biol. Chem. 270(45), 27244-27253 (1995)

35. Vandekerckhove, F., Schenkman, S., Pontes de Carvalho, L., Tomlinson, S., Kiso, M., Yoshida, M., Hasegawa, A., Nussenzweig, V.: Substrate specificity of the Trypanosoma cruzi trans-sialidase. Glycobiology 2(6), 541-548 (1992)

36. Scudder, P., Doom, J.P., Chuenkoval, M., Manger, I.D., Pereira, M.E.A.: Enzymatic characterization of beta-D-galactoside alpha 2,3-trans-sialidase from Trypanosoma cruzi. J. Biol. Chem. 268(13), 9886-9891 (1993)

37. Schenkman, S., Ferguson, M.A.J., Heise, N., de Almeida, M.L., Mortara, R.A., Yoshida, N.: Mucin-like glycoproteins linked to the membrane by glycosylphosphatidylinositol anchor are the major acceptors of sialic acid in a reaction catalyzed by trans-sialidase in metacyclic forms of Trypanosoma cruzi. Mol. Biochem. Parasitol. 59(2), 293-303 (1993)

38. Schenkman, S., Eichinger, D.: Trypanosoma cruzi trans-sialidase and cell invasion. Parasitol. Today 6(6), 218-222 (1993)

39. Tomlinson, S., Pontes de Carvalho, L.C., Vandekerckhove, F., Nussenzweig, V.: Role of sialic acid in the resistance of Trypanosoma cruzi trypomastigotes to complement. J. Immunol. 153(7), 3141-3147 (1994)

40. Buscaglia, C.A., Campo, V.A., Frasch, A.C.C., Di Noia, J.M.: Trypanosoma cruzi surface mucins: host-dependent coat diversity. Nat. Rev. Microbiol. 4(3), 229-236 (2006)

41. Salazar, N.A., Mondragon, A., Kelly, J.M.: Mucin-like glycoprotein genes are closely linked to members of the trans-sialidase super-family at multiple sites in the Trypanosoma cruzi genome. Mol. Biochem. Parasitol. 78(1-2), 127-136 (1996)

42. Schenkman, S., Jiang, M.S., Hart, G.W., Nussenzweig, V.: A novel cell surface trans-sialidase of Trypanosoma cruzi generates a stagespecific epitope required for invasion of mammalian cells. Cell 65(7), 1117-1125 (1991)

43. Brener, Z.: Biology of Trypanosoma cruzi. Annu. Rev. Microbiol. 27, 347-382 (1973)

44. Yoshida, N.: Molecular basis of mammalian cell invasion by Trypanosoma cruzi. An. Acad. Bras. Cienc. 78(1), 87-111 (2006)

45. Fernandes, M.C., Andrews, N.W.: Host cell invasion by Trypanosoma cruzi: a unique strategy that promotes persistence. FEMS Microbiol. Rev. 36(3), 734-747 (2012)

46. Camargo, M.M., Almeida, I.C., Pereira, M.E., Ferguson, M.A.J., Travassos, L.R.: Glycosylphosphatidylinositol-anchored mucinlike glycoproteins isolated from Trypanosoma cruzi trypomastigotes initiate the synthesis of proinflammatory cytokines by macrophages. J. Immunol. 158(12), 5890-5901 (1997)

47. Yoshida, N., Dorta, M.L., Ferreira, A.T., Oshiro, M.E., Mortara, R.A., Acosta-Serrano, A., Favoreto Júnior, S.: Removal of sialic acid from mucin-like surface molecules of Trypanosoma cruzi metacyclic trypomastigotes enhances parasite-host cell interaction. Mol. Biochem. Parasitol. 84(1), 57-67 (1997) 
48. Ruiz, R.C., Rigoni, V.L., Gonzalez, J., Yoshida, N.: The 35/50 kDa surface antigen of Trypanosoma cruzi metacyclic trypomastigotes, an adhesion molecule involved in host cell invasion. Parasite Immunol. 15(2), 121-125 (1993)

49. Yoshida, N., Mortara, R.A., Araguth, M.F., Gonzalez, J.C., Russo, M.: Metacyclic neutralizing effect of monoclonal antibody 10D8 directed to the 35- and 50-kilodalton surface glycoconjugates of Trypanosoma cruzi. Infect. Immun. 57(6), 1663-1667 (1989)
50. Ruiz, R.C., Favoreto Jr., S., Dorta, M.L., Oshiro, M.E., Ferreira, A.T., Manque, P.M., Yoshida, N.: Infectivity of Trypanosoma cruzi strains is associated with differential expression of surface glycoproteins with differential $\mathrm{Ca} 2+$ signaling activity. Biochem. J. 330(1), 505-511 (1998)

51. Mortara, R.A., Silva, S., Araguth, M.F., Blanco, S.A., Yoshida, N.: Glycoconjugates of Trypanosoma cruzi metacyclic trypomastigotes. Infect. Immun. 60(11), 4673-4678 (1992) 